\title{
Performance Measures of Tele-Protection System Based on Networked Microwave Radio Link
}

\author{
Jitendra Singh \\ Assistant Professor (Senior Grade), Department of Computer Science and Engineering, SRM University, Delhi NCR \\ Campus, India (Research Scholar, NIMS University, Jaipur, Rajasthan, India) \\ jitendra.jit@gmail.com
}

Rabins Porwal

Associate Professor, Department of IT, ITS, Mohan Nagar, Ghaziabad

rabinsp@ieee.org

\author{
S.P.Singh \\ Associate Professor, Department of Computer Science and Engineering NIMS University, \\ Jaipur, Rajasthan, India
}

\begin{abstract}
The purpose of paper is to compute reliability of Digital Tele-Protection System employing Digital Microwave Radio Link. In this paper, the whole TeleProtection system is divided into six subsystems. The first subsystem is digital relaying equipment. Second subsystem is the tale-protection signaling equipment where the timing to sample line power currents is synchronized. Both relaying and Tele-Protection signaling equipments, are now assemble three-phase current data, sampled at $600 \mathrm{~Hz}$ for $50 \mathrm{~Hz}$ EHV systems and some control data into a 90 bit frame format at a transmission rate of $54 \mathrm{~Kb} / \mathrm{s}$. A frame consists of a frame synchronization field and some information fields including a cycle's redundancy check field. The third subsystem, Tele-Protection signed multiplexing equipment generates a super frame by multiplexing the seven, $54 \mathrm{~Kb} / \mathrm{s}$ Tele-Protection signals into a $1.544 \mathrm{Mb} / \mathrm{s}$ stage using multi-point sampling technique to minimize the delay time variation and send them to the subsystem four, which is radio equipment, from here the data is transmitted with the help of the channel (subsystem 5) to the repeater station (subsystem 6). The subsystem six is used to remove the noise and to improve the power of the data. In this paper, we have taken standby digital relaying equipment and that can be online through an imperfect switching device. Also parallel redundant Tele-Protection signaling equipment is taken to improve system's overall performance.
\end{abstract}

Index Terms - Reliability, MTTF, Tele-Protection system.

\section{INTRODUCTION}

Any reliability study essentially remains an academic exercise unless due consideration is given to economic factors and organizational feasibility. From a practical viewpoint, it is the ability to achieve incremental improvement in system reliability that is more important than maximum reliability. As shown in figure 1, a greater investment in reliability improvement in the earlier stages is generally expected to reduce maintenance costs and investments in spares inventories.

The trade-off between desired reliability levels and practical limiting factors such as unreliability of parts preclude $100 \%$ system reliability. The objective should then move from achieving maximum reliability levels to those that are both feasible and mutually acceptable to the producer and consumer alike. The concept of assigning a quantitative basis for failure control is particularly important, so as to plan for, specify and achieve the optimum level of reliability in practical applications. Studies have indicated that the total cost of support of low quality equipment over the life of the device is often times more than the initial acquisition cost.

\section{RELATED WORK}

Digital Tele-Protection systems such as microprocessor-based PCM, current differential relaying systems employing microwave or fiber optic links as telecommunication media have been widely used while fiber optics. Which have the advantage of immunity to electromagnetic interference, high quality and wide-band transmission are increasing by applied to Tele-Protection and control systems, microwave radio systems are still highly reliable transmission media for those purpose. Microwave systems are rapidly being digitized. With respect to digital radio systems, their transmission performance requirements should be examined when employing dedicated digital microwave links for teleprotection. It should be noted that Tele-Protection systems are more stringent than ordinary communication systems such as computer communication systems or file transfer systems which allowed the certain number of transmission delay increase resulting from requested repeat transmission. For power system relaying engineers as well as communication ones it is meaningful and 
urgent to discuss such issues related to interface and connectivity between relaying and Tele-Protection equipments, since relaying equipments are taking in communication functions while Tele-Protection equipments have been mainly designed to meet a relatively severe reliability requirement for teleprotection.

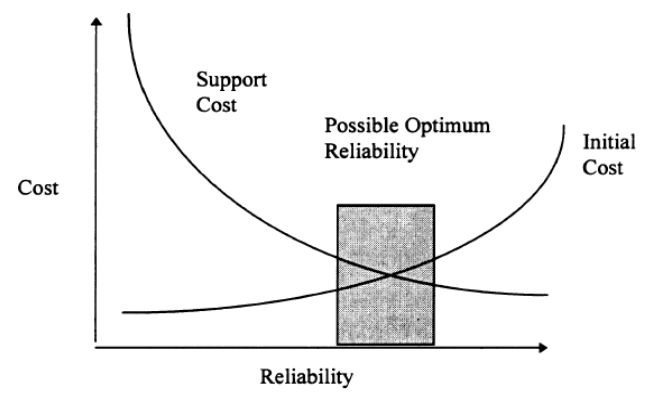

Fig-1 Cost verses reliability trade-off

\section{A. Definitions of reliability}

A current fashionable alternative view of reliability attempts to analyze the physics of the failure process and, through a mathematical model, determine the time to failure. This approach requires knowledge of the failure mechanisms [1-3] and the basic causes of failure. Mean time to failure is determined on the basis of known or predicted stresses, environmental factors, operating conditions, material properties and geometries.

In our daily life, we feel that things fail. During the past two years this author has experienced that a washing machine fails, a car battery becomes dead, a toaster oven electrical plug burns, water-heater leaks, a floppy disk drive damages, a TV remote control quit functioning, a stereo amplifier quit, an automobile engine starter fail, and a house roof leaks. The reason of failure of washing machine motor, the car battery and the engine starter was the result of experienced wear out after a normal life.

The toaster oven plug was a poor design [5,6], considering the amount of current passing through it. Corrosion of the hot water tank caused it to break. The corrosion was partly attributed to the lack of preventive maintenance, which required periodical draining and cleaning of the bottom of the tank. The failure of the disk drive was a result of an unknown (premature) mechanical failure, and the TV remote controls failure was caused by a "random" electronic component failure. On the other hand, the stereo amplifier failure was caused by an open at a solder joint. Poor construction resulted in the house roof leaking adjacent to the dormers.

Some of these failures caused much inconvenience in addition to their economic impact. Several of the failure raised concerns about personal safety, although no injuries resulted from them. Some standard definitions of reliability are as given below:

Definition 1: Radio Electronics Television Manufactures Association (RETMA)-1955
"Reliability is the probability [4] of a device performing its purpose adequately for the period of time intended under the operating conditions encountered". This definition of reliability is considered to be standard one and is widely accepted by most contemporary reliability authorities. This definition lays stress on four important factors namely, probability, adequate performance, time and operating conditions.

- Probability: It is quantitative measure of an event out of the total number of trials.

- Adequate Performance: It indicates the criteria for operation of the device to be satisfactory.

- Time: It is the period during which one can expect the satisfactory performance.

- Operating conditions: The terms used in the operation of a device called operating conditions. Temperature, humidity shocks and vibrations, etc., are the terms of the operation for a device.

\section{Definition 2: HOSFORD (1960)}

"Dependability is the probability that the system will be able to operate when needed". [9] Thus, HOSFORD defined reliability through the concept of dependability. According to him four important measures of dependability are as follows

- Point wise - availability or Operational readiness: It is the probability that the system will be able to operate within the tolerances at a given instance of time. The term, 'availability' has been used by 'Welker' and 'Horne' (1960) for this quantity.

- Reliability: It is the probability that the system will be able to operate without a failure for a given interval of time. Failure is defined as the inability to operate within tolerances.

- Interval availability or Efficiency: It is the expected fraction of given interval of time that the system will be able to operate within tolerances.

- Interval Reliability: It is defined as the probability that at a specified time the system is operating and remains operative for the duration $\mathrm{t}$ to $t+x$ Symbolically, interval reliability is

$$
R(t, x)=P[\times(\mu)=1, \forall u, t \leq \mu \leq t+x]
$$

\section{Definition 3: GAYANA (1961)}

"Reliability is the probability of performance without failure of specified function under given conditions for a specific period".

\section{Definition 4: GAVER (1963)}

"Reliability is a function of environment as well as the component itself and particularly depends upon environmental variability".

Definition 5: POLOVKO (1968) 
"Reliability is the ability of equipment to preserve its output characteristics (parameters) within established limits under given operating conditions".

From all these definitions, qualitatively; reliability is the probability that a device will operate as anticipated. Quantitatively reliability is given by

$$
R(t)=P(T>t), \quad 0 \leq t<\infty
$$

where ' $\mathrm{T}$ ' is the time of failure to the device, ( $\mathrm{T}$ itself is variable) and $t$ is any time. Generally, initially the device is assumed to be working normally and every device definitely suffers failure. Therefore, symbolically.

$$
R(0)=1 \text { and } \lim _{t \rightarrow \infty} R(t)=0
$$

\section{B. Reliability function}

Reliability is defined as the probability that a system (component) will function over some period of time t. To express this relationship mathematically [11] we define the continuous random variable $\mathrm{T}$ to be the time to failure of the system (component); $T \geq 0$. Then reliability can be expressed as

$$
R(t)=\operatorname{Pr}\{T \geq t\}
$$

where $R(t) \geq 0, R(0)=1$ and $\lim _{t \rightarrow \infty} R(t)=0$.

For a given value of $\mathrm{t}, R(t)$ is the probability that the time to failure is greater than or equal to $t$.

If we define

$$
F(t)=1-R(t)=\operatorname{Pr}(T<t)
$$

where $\mathrm{F}(0)=0$ and $\lim _{t \rightarrow \infty} F(t)=1$

then $F(t)$ is the probability that a failure occurs before time $t$. We will refer to $R(t)$ as the reliability function and $F(t)$ as the cumulative distribution function (CDF) of the failure distribution.

A third function, defined by

$$
f(t)=\frac{d F(t)}{d t}=-\frac{d R(t)}{d t}
$$

It is called the probability density function (PDF). This function describes the shape of the failure distribution.

The PDF, $f(t)$, has following two properties

$$
f(t) \geq 0 \text { and } \int_{0}^{\infty} f(t) d t=1
$$

Given the PDF, $f(t)$ then

$$
\begin{aligned}
F(t) & =\int_{0}^{t} f\left(t^{\prime}\right) d t^{\prime} \\
\text { and } R(t) & =\int_{t}^{\infty} f\left(t^{\prime}\right) d t^{\prime}
\end{aligned}
$$

\section{Mean time to failure}

The mean time to failure (MTTF) is defined by

$$
M T T F=\int_{0}^{\infty} R(t) d t
$$

The concepts of reliability engineering and probability theory attain significantly complex levels when applied to complex systems/ networks. This is because modern electronic systems are inherently complex due to their technology and the networks of devices inside them. Moreover, electronic parts are particularly sensitive to a multitude of external and internal stresses that drastically alter their behavior.

Each part in a system may have one or more potential modes of failure and each mode may have one or more failure mechanisms. This complexity of component reliability carries over to the reliability of the system that houses the components. It behooves engineers therefore, to be extremely careful in conducting a quantitative and qualitative analysis of the reliability of such complex systems.

Even with rough estimation techniques such as population analysis, resource dedication to evaluate reliability aspects of a system increases as the complexity of the system increases. Garg R.C. (1963) made use of reliability evaluation techniques for the first time to tackle general distribution in the reliability context. He introduced the concept of 'reduced efficiency', which results from a practical situation, where a complex system goes on operating (of course, with lesser efficiency). Even after the failure of some less important components of the system. The idea was further developed by Govil, A.K. and Gupta, P.P., who incorporated the concept of three repair disciplines, viz., Head-of-line, Pre-emptiveresume, and Pre-emptive-repeat. But still there remain a gap to fill in order to make the models more practical and accurate. In the above work negligible care was given to failures due to logical errors, and structural redundancy. Thus in this study, we have included these factors, in order to find more accurate results.

\section{Relevance of the Bathtub Curve to Part Failure Rates}

"The time between successive failures is a continuous random quantity". From a probabilistic viewpoint, if the distribution function modeling the variable of interest is known, this random variable can be analyzed using a failure model that is based on given conditions. 
Probability theory provides the relationship between these failure models and life test results of failure rates. The distribution shown in figure- 2 is widely applied in the scientific community to model the failure distribution of most electronic parts.

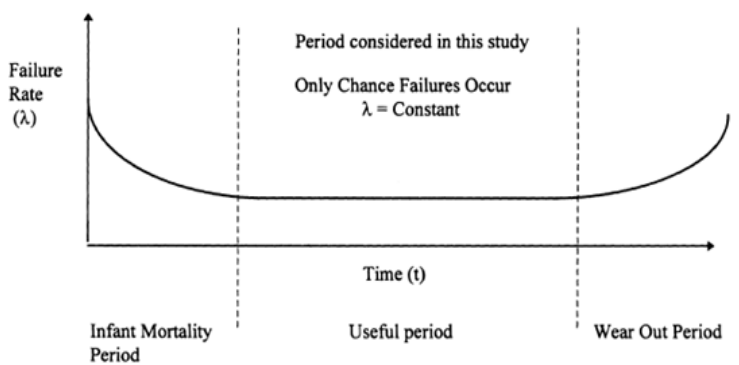

Fig-2: Bathtub curve depicting the failure rate of electronic components

This distribution, commonly known as the bathtub curve can be stated as the sum of three basic distributions as stated in Equation-11:

$$
f(t)=W 1 f l(t)+W 2 f 2(t)+W 3 f 3(t)
$$

where $\mathrm{W} 1, \mathrm{~W} 2$, and $\mathrm{W} 3$ are adjustable weight factors that facilitate the combination of the three distributions such that $\mathrm{f}(\mathrm{t})$ satisfies the definition of a probability distribution function. In our relevant analysis, W1 +W2 $+\mathrm{W} 3=1$.

The hazard rate in the infant mortality period is initially high and is expected to decrease rapidly with time. This phenomenon occurs mainly because of flaws incorporated during the manufacturing process that escape quality control checks and cause the part to fail.

High failure rate units may also be identified in this region. It has been observed that the failure distribution in this period represented by $\mathrm{f}(\mathrm{t})$ in equation -11 , may be approximated by the gamma distribution described by equation-12.

$$
f_{1}(t)=\frac{1}{b \Gamma(a)} \frac{t^{(a-1)}}{b^{(a-1)}} e^{(-t b)} \quad \text { for } t>0
$$

Where,

$\Gamma$ (a) is the gamma function and is equal to (a-1)!, when (a-1) is a positive integer.

$\mathrm{b}=\mathrm{MTBF}$ of the whole quipment and, $a=$ Number of failiures.

It may be noted that the characteristic for a $<1$ mimics the burn-in period of the bathtub curve, and for $a=1$, i.e. corresponding to time to first failure, equation-12 reduces to the exponential distribution described by equation-13. The work done in this study assumes that all parts have passed this burn-in period and have matured into the constant failure rate period. This constant failure rate period is the useful operating life of the part or equipment, and reflects the period in which the measurement of equipment reliability is most relevant in the electronics industry. Failures of parts and systems are essentially random in this interval ensuring that the failure rate is essentially constant as shown in figure-3

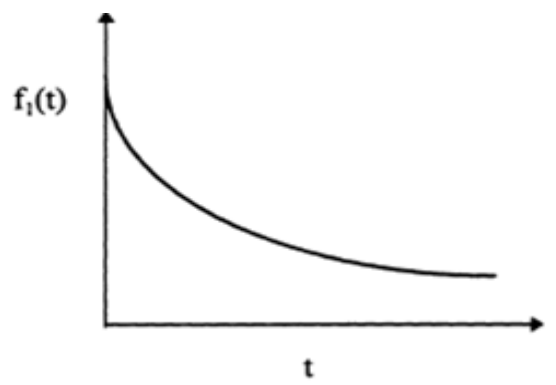

Fig-3: Graph depicting the exponential failure distribution

It has been observed that the failure distribution in this period represented by $f-2(t)$ in equation- 13 .

$$
f_{2}(t)=\lambda e^{-\lambda t}
$$

where $\lambda$ is the constant failure rate, $\theta=1 / \lambda$ is the Mean Time Between Failures (MTBF) defined as the arithmetic average of the failure free intervals for non-repairable systems.

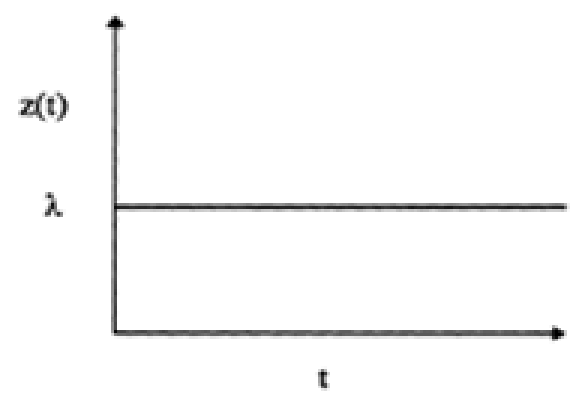

Fig-4: Graph depicting a constant failure rate for the exponential time distribution

The assumption of an exponential distribution of failures in the useful operating life of electronic equipment is a very important one for the work done in this thesis. As described later, this assumption allows the failure rate of large non-redundant systems to be expressed as the sum of constituent part failure rates by using product rule of reliability.

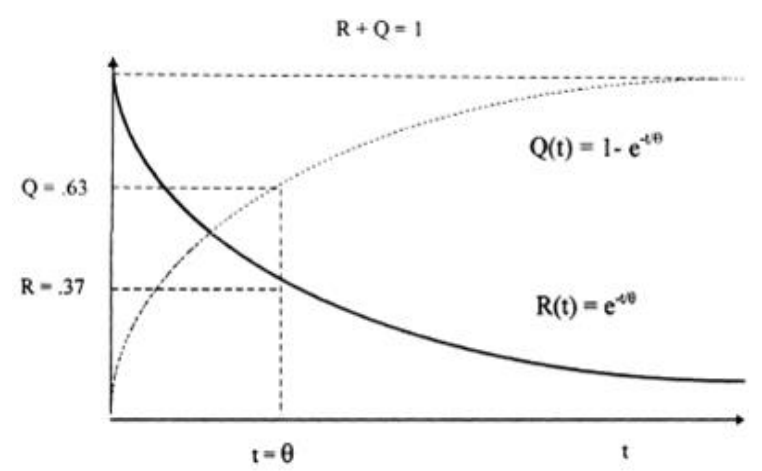

Fig-5: Reliability and unreliability curves for a constant failure rate 
In order to analyze the parallel configurations used in this research work, another measure of system performance needs to be introduced. Just as reliability can be defined as the probability of successful operation for time ' $t$ ', so can another measure of system performance called unreliability be defined. It is the probability of failure for the time interval ' $t$ '. Clearly, the probability of either failure or success is equal to 1. i.e.

$$
\mathrm{R}(\mathrm{t})+\mathrm{Q}(\mathrm{t})=1
$$

for operating time $\mathrm{t}$, where $\mathrm{R}(\mathrm{t})$ is the reliability and $\mathrm{Q}(\mathrm{t})$ is the unreliability. For a constant failure rate,

$$
\begin{gathered}
\mathrm{R}(\mathrm{t})=e^{-t / \theta} \\
\mathrm{Q}(\mathrm{t})=1-e^{-t / \theta}
\end{gathered}
$$

Figure 5, shows the reliability and unreliability curves for a constant failure rate. The Poisson distribution is also an extremely useful distribution in the reliability prediction of electronic equipment. It characterizes rare events and represents the probability of an event occurring a given number of times in a given interval of time. It can be seen from equation-17 that the exponential distribution is only a special case of the Poisson distribution, specifically when considering the probability of the first failure. Assuming that the total number of operating parts remains the same, the Poisson distribution can be described as

$$
f(x)=\frac{e^{-\lambda t}(\lambda t)^{X}}{x !}
$$

The bath tub failure rate curve has been widely acknowledged by scientists as an excellent indicator of the reliability behavior of electronic parts and systems. Depending upon the life cycle stage of observation and the data gathered, this behavior can be represented by various other probability distributions. Many of these distributions are theoretically relevant in reliability mathematical modeling. In practice the exponential, Poisson, Raleigh, Normal, Gamma and Weibull distributions are the probability distributions that are commonly employed to model the reliability of electronic components.

The remaining paper is organized as follows: In Section II, we describe the Block diagram of TeleProtection System used, In Section III, we describe the Formulation of Mathematical Model. In Section IV results obtained are discussed. And in Section V, we conclude our paper.

\section{MATERIAL \& METHODS}

Using algebra of logics, mathematical formulation of the system has been given and solved. This mathematical model has been solved by employing Boolean function technique. Reliability of considered system has been computed. Reliability functions have been obtained in two different cases, when failures follow weibull and exponential time distributions.

An important reliability parameter, viz. mean time to failure of considered system has also been computed to improve practical utility of the model. Graphical illustration followed by a numerical computation has been appended at the end to highlight important results of the study.

The following assumptions have been associated with this model:

1. Initially, all components of the considered system are good.

2. The reliability of the constituent components of the system is known in advance.

3. The states of all components are S-independent.

4. Failure rates of all components are arbitrary.

5. There is no repair facility.

6. The states of each component and the whole system is either good (operating) or bad (failed).

7. Switching device used to online standby digital relaying equipment is imperfect.

\begin{tabular}{|c|c|c|}
\hline$x_{1}, x_{3}$ & : & State of digital relaying equipment. \\
\hline$x_{2}$ & & State of switching device. \\
\hline$x_{4}, x_{5}$ & & $\begin{array}{l}\text { States of Tele-Protection signaling } \\
\text { equipment. }\end{array}$ \\
\hline$x_{6}$ & & $\begin{array}{l}\text { State of Tele-Protection signal } \\
\text { multiplexing equipment. }\end{array}$ \\
\hline$x_{7}$ & & States of digital radio equipment. \\
\hline$x_{8}$ & & State of radio propagation path. \\
\hline$x_{9}$ & & State of repeater station. \\
\hline$\wedge / \vee$ & : & Conjunction/Disjunction. \\
\hline
\end{tabular}

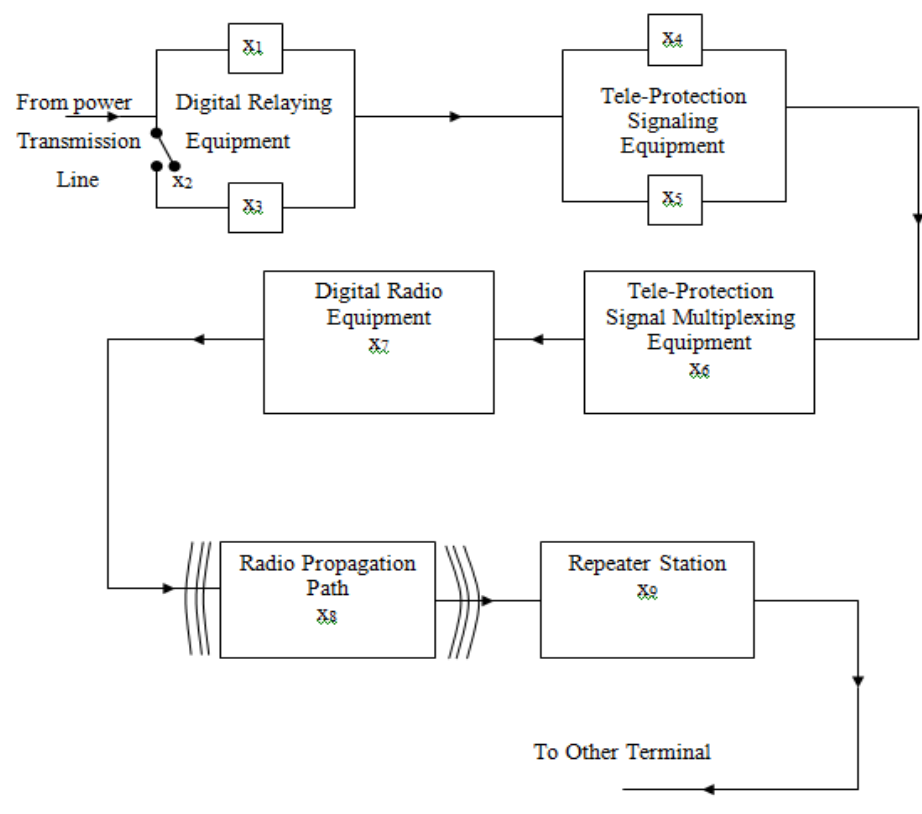

Fig- 6: Block diagram of Tele-Protection System

List of notations is as follows: 


$$
\begin{aligned}
& x_{i}^{\prime} \quad: \quad \text { Negation of } x_{i}, \forall i=1,2,--9 . \\
& x_{i}:\left\{\begin{array}{l}
0 \text { in bad state } \\
1 \text { in good state }
\end{array}, \forall i=1,2,--9\right. \\
& \operatorname{Pr}(F=1) \quad \text { : Probability of successful operation } \\
& \text { of the function } F \text {. } \\
& R_{S} \quad: \text { Reliability of the system as a whole. } \\
& R_{i} \quad: \text { Reliability of the component }
\end{aligned}
$$

\section{FORMULATION OF MATHEMATICAL MODEL}

Conditions of capability for successful operation of considered system, in terms of logical matrix, can be expressed as given below:

$$
F\left(x_{1}, x_{2}--x_{9}\right)=\left[\begin{array}{ccccccc}
x_{1} & x_{4} & x_{6} & x_{7} & x_{8} & x_{9} & \\
x_{1} & x_{5} & x_{6} & x_{7} & x_{8} & x_{9} & \\
x_{2} & x_{3} & x_{4} & x_{6} & x_{7} & x_{8} & x_{9} \\
x_{2} & x_{3} & x_{5} & x_{6} & x_{7} & x_{8} & x_{9}
\end{array}\right]
$$

Reliability of considered system as a whole is given by:

$$
\begin{gathered}
R_{S}=\operatorname{Pr}\left\{F\left(x_{1}, x_{2}--x_{9}\right)=1\right\} \\
=R_{6} R_{7} R_{8} R_{9}\left[R_{1} R_{4}+S_{4} R_{1} R_{5}+S_{1} R_{2} R_{3} R_{4}+S_{1} S_{4} R_{2} R_{3} R_{5}\right]
\end{gathered}
$$

where $R_{i}$ is the reliability of component corresponding to state $x_{i}$ and $S_{i}=1-R_{i} \quad \forall \mathrm{i}=1,2---9$.

$$
\begin{aligned}
\therefore R_{S}= & R_{6} R_{7} R_{8} R_{9}\left[R_{1} R_{4}+R_{1} R_{5}+R_{2} R_{3} R_{4}+R_{2} R_{3} R_{5}+R_{1} R_{2} R_{3} R_{4} R_{5}\right. \\
& \left.-R_{1} R_{4} R_{5}-R_{1} R_{2} R_{3} R_{4}-R_{1} R_{2} R_{3} R_{5}-R_{2} R_{3} R_{4} R_{5}\right]
\end{aligned}
$$

\section{A. Some Particular Cases}

Case $i$ : When reliability of each component is $R$ :

In this case, putting $R_{i}(\mathrm{i}=1,2-----9)=\mathrm{R}$ in equation (20), we get

$$
R_{S}=2 R^{6}+R^{7}-3 R^{8}+R^{9}
$$

Case ii: When failures follow Weibull time distribution:

Let $\lambda_{i}$ be the failure rate of component corresponding to state $x_{i}$ of the system and it follows weibull time distribution, then

$$
R_{i}=\exp \left\{-\lambda_{i} t^{\alpha}\right\}
$$

where $\alpha$ is a real positive parameter.

Putting this value in equation (20), we obtain

$$
\mathrm{R}_{\mathrm{SW}}(t)=\sum_{i=1}^{5} \exp \cdot\left\{-a_{i} t^{\alpha}\right\}-\sum_{j=1}^{4} \exp \cdot\left\{-b_{j} t^{\alpha}\right\}
$$

where $\alpha$ is some real positive parameter and

$$
\begin{aligned}
& a_{1}=c+\lambda_{1}+\lambda_{4} \\
& a_{2}=c+\lambda_{1}+\lambda_{5} \\
& a_{3}=c+\lambda_{2}+\lambda_{3}+\lambda_{4} \\
& a_{4}=c+\lambda_{2}+\lambda_{3}+\lambda_{5} \\
& a_{5}=c+\lambda_{1}+\lambda_{2}+\lambda_{3}+\lambda_{4}+\lambda_{5} \\
& b_{1}=c+\lambda_{1}+\lambda_{4}+\lambda_{5} \\
& b_{2}=c+\lambda_{1}+\lambda_{2}+\lambda_{3}+\lambda_{4} \\
& b_{3}=c+\lambda_{1}+\lambda_{2}+\lambda_{3}+\lambda_{5} \\
& b_{4}=c+\lambda_{2}+\lambda_{3}+\lambda_{4}+\lambda_{5} \\
& c=\lambda_{6}+\lambda_{7}+\lambda_{8}+\lambda_{9}
\end{aligned}
$$

Also, an important reliability parameter, namely mean time to failure (MTTF), in this case, is given by

$$
\begin{gathered}
\operatorname{MTTF}(W)=\int_{0}^{\infty} R_{S W}(t) d t \\
=\frac{\Gamma\left(\frac{1}{\alpha}\right)}{\alpha}\left[\sum_{i=1}^{5} \frac{1}{a_{i}^{1 / \alpha}}-\sum_{j=1}^{4} \frac{1}{b_{j}^{1 / \alpha}}\right]
\end{gathered}
$$

Case iii: When failures follow exponential time distribution:

Exponential time distribution is a particular case of Weibull time distribution for $\alpha=1$ and is very useful in numerous practical problems. So, putting $\alpha=1$ in equation (22), we get

$$
\mathrm{R}_{\mathrm{SE}}(t)=\sum_{i=1}^{5} \exp \cdot\left\{-a_{i} t\right\}-\sum_{j=1}^{4} \exp \cdot\left\{-b_{j} t\right\}
$$

and

$$
\begin{gathered}
\text { M.T.T.F. }(E)=\int_{0}^{\infty} R_{S E}(t) d t \\
=\sum_{i=1}^{5} \frac{1}{a_{i}}-\sum_{j=1}^{4} \frac{1}{b_{j}}
\end{gathered}
$$


where $a_{i}^{\prime} s$ and $b_{j}^{\prime} s$ have been mentioned earlier.

\section{B. Numerical computation}

Let us consider

(i) $\lambda=0.001, \alpha=2$ and $\mathrm{t}=0,1,2,----10$. Using these values in equation (21), we compute table- 1 .

(ii) $\lambda=0.001$, and $t=0,1,2,----10$. Using these values in equation (23), we compute table-1.

(iii) $\lambda=0,0.01,0.02,---0.10$ and $\alpha=2$. Using these values in equation (22), we compute table- 2 .

(iv) $\lambda=0,0.01,0.02,---0.10$. Using these values in equation (24), we compute table-2.

Table-1: RSW(t) and RSE(t)

\begin{tabular}{|c|c|c|}
\hline $\mathrm{T}$ & $\mathrm{R}_{\mathrm{SW}}(\mathrm{t})$ & $\mathrm{R}_{\mathrm{SE}}(\mathrm{t})$ \\
\hline 0 & 1 & 1 \\
\hline 1 & 0.996005 & 0.996005 \\
\hline 2 & 0.98408 & 0.99202 \\
\hline 3 & 0.964409 & 0.988045 \\
\hline 4 & 0.9373 & 0.98408 \\
\hline 5 & 0.903197 & 0.980126 \\
\hline 6 & 0.862681 & 0.976181 \\
\hline 7 & 0.816472 & 0.972247 \\
\hline 8 & 0.765423 & 0.968323 \\
\hline 9 & 0.710507 & 0.964409 \\
\hline 10 & 0.652791 & 0.960505 \\
\hline
\end{tabular}

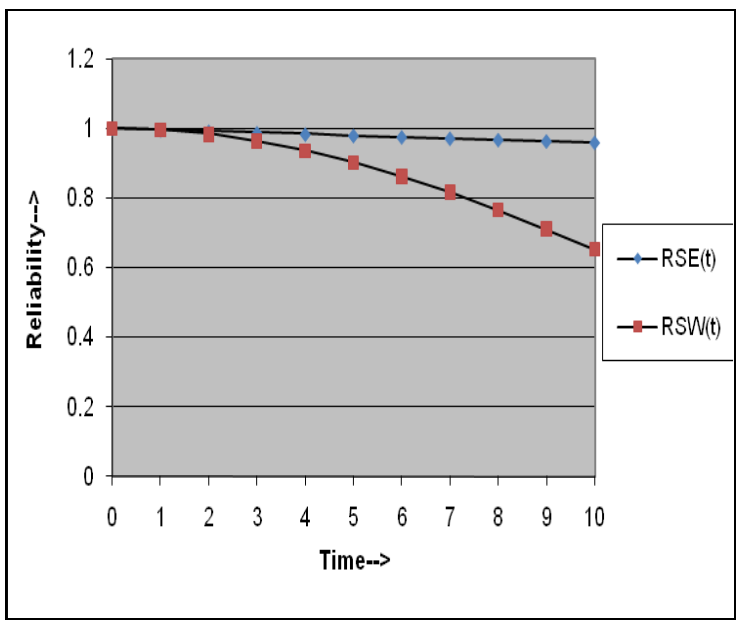

Fig-7: Reliablity vs Time
Table-2 MTTF(W) and MTTF(E)

\begin{tabular}{|c|c|c|}
\hline$\lambda$ & MTTF(W) & MTTF(E) \\
\hline 0 & $\infty$ & $\infty$ \\
\hline 0.01 & 21.2302 & 4.13986 \\
\hline 0.02 & 10.6151 & 2.927323 \\
\hline 0.03 & 7.076733 & 2.390149 \\
\hline 0.04 & 5.30755 & 2.06993 \\
\hline 0.05 & 4.24604 & 1.851402 \\
\hline 0.06 & 3.538367 & 1.690091 \\
\hline 0.07 & 3.032886 & 1.56472 \\
\hline 0.08 & 2.653775 & 1.463662 \\
\hline 0.09 & 2.358911 & 1.379953 \\
\hline 0.1 & 2.12302 & 1.309139 \\
\hline
\end{tabular}

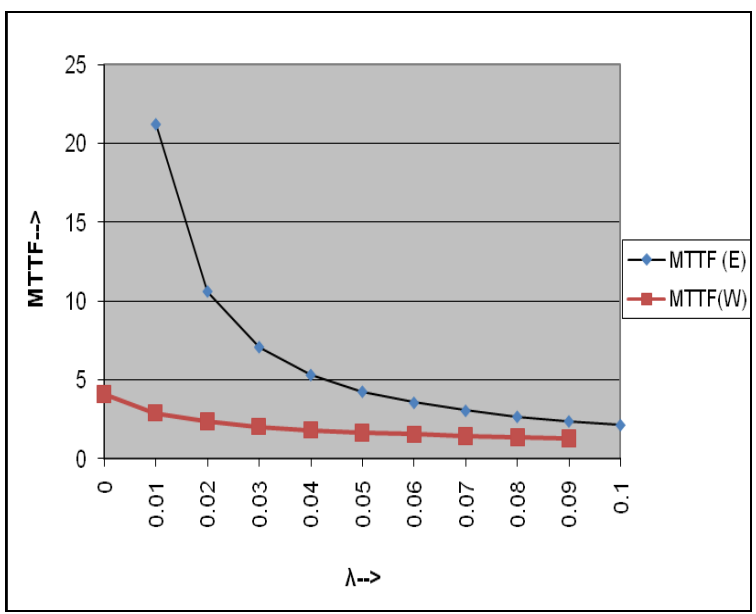

Fig-8 MTTF vs $\lambda$

\section{RESULTS AND DISCUSSION}

The graph of table-1 has shown in fig-7. Analysis of table-1 and fig-7 reveals that reliability function RSW(t) decreases catastrophically in the beginning but thereafter it decreases constantly. The value of $\mathrm{RSE}(\mathrm{t})$ remains better as compared of RSW(t). The graph of table- 2 has shown in fig- 8 . A critical examination of table- 2 and fig8 yields that the value of $\operatorname{MTTF}(\mathrm{E})$ decreases rapidly as we make increase in the values of failure rate $\lambda$ but thereafter it decreases in a constant manner. The value of $\operatorname{MTTF}(\mathrm{E})$ remains better as compared of $\operatorname{MTTF}(\mathrm{W})$. 


\section{CONCLUSIONS}

Using algebra of logics, we have done mathematical formulation of the system. This mathematical model has been solved by employing Boolean function technique. Reliability of considered system has been computed. Reliability functions have been obtained in two different cases, when failures follow weibull and exponential time distributions.

An important reliability parameter, viz. mean time to failure of considered system has also been computed to improve practical utility of the model. Graphical illustration followed by a numerical computation has been appended at the end to highlight important results of the study. The study of analysis of reliability can help in increasing the quality of the Tele-Protection system. To ensure the system performance throughout its life, it is necessary to set up proper maintenance planning and control which can be done after studying the variation of reliability with respect to time.

\section{REFERENCES}

[1] Agnihotri, R.R., Satsangi, S. K., Two Non-identical Unit System with Priority Based Repair and Inspection, Microelectronic Reliab. , Vol. 36 (2), pp 279 - 282, 1996.

[2] Cassady, C.R., Lyoob, I.M., Schneider, K., Pohi, E. A., A Generic Model of Equipment Availability under Imperfect Maintenance, IEEE TR on Reliability, Vol.54, issue - 4, pp $564-571,2005$.

[3] Chung, W. K., A k-out -of n, G Redundant System with the Presence of Chance with Multiple Critical Errors, Microelectronic Reliab., Vol. 33, pp 334-338, 1993.

[4] Cluzeau, T., Keller, J., Schneeweiss, W., An Efficient Algorithm for Computing the Reliability of Consecutive-kout-of-n:F Systems, IEEE TR. on Reliability, Vol.57 (1), pp 84-87, 2008.

[5] Dhillon, B. S. and Yang N. J.: Stochastic Analysis of Standby System with Common Cause Failure and Human Error, Microelectronic Reliab., Vol. 32, pp 1699 - 1712, 1992.
[6] Dhillon, B. S., Reliability Analysis of Non-Maintained Parallel System Subject to Hardware Failure and Human Error, Microelectronic Reliab. Vol. 25, pp 121 -122, 1985.

[7] Fang - Ming, S., Xuemin, S., Pin - Han, H., Reliability Optimization of Distributed Access Networks with Constrained Total Cost, IEEE TR on Reliability, Vol.54, issue - 3, pp 421 - 430, 2005.

[8] Gutierrez, P.H., Aguirre, T. V., Christen, J. A., A Practical Method for Obtaining Prior Distributions in Reliability, IEEE TR. on Reliability, Vol. 54, issue - 2, pp 262 - 269, 2005.

[9] Kijima, M., Markov Processes for Stochastic Modeling, CRC Press, 1997.

[10] Lindsey, J.K., Statistical Analysis of Stochastic Processes in Time, Cambridge University Press, 2004.

[11] Linkov, L.N., Asymptotic Statistical Methods for Stochastic Processes, American Mathematical Society, 2001.

[12] Nagraja, H.N., Kannan, N., Krishnan, N.B., Reliability, Springer Publication, 2004.

Jitendra Singh, Ph.D. candidate in NIMS University, Jaipur Rajasthan, India. His major field of study is Software Engineering and Reliability. At present, he is working as Assistant Professor (Senior Grade) in the Department of Computer Science and Engineering, SRM University, Delhi NCR Campus, Ghaziabad, India.

Rabins Porwal, Ph.D. and Associate Professor in the Department of IT, Institute of Technology and Science (ITS), Ghaziabad (UP), India. His major field of study is Software Engineering, Computer Networks.

S. P. Singh, Ph.D., Associate Professor and Head of Department of Computer Science and Engineering, NIMS University, Jaipur, Rajasthan, India. His major field of study is Software Engineering, Computer Networks.

How to cite this paper: Jitendra Singh, Rabins Porwal, S.P.Singh,"Performance Measures of Tele-Protection System Based on Networked Microwave Radio Link", IJCNIS, vol.6, no.5, pp.21-28, 2014. DOI: 10.5815/ijcnis.2014.05.03 